\title{
Generalized contractions in metric spaces endowed with a graph
}

\section{Cristian Chifu* and Gabriela Petruşel}

${ }^{*}$ Correspondence:

Cristian.Chifu@tbs.ubbcluj.ro

Department of Business,

Babeş-Bolyai University

Cluj-Napoca, Horea street, No.7,

Cluj-Napoca, Romania

\begin{abstract}
A very interesting approach in the theory of fixed points in some general structures was recently given by Jachymski (Proc. Amer. Math. Soc. 136:1359-1373, 2008) and Gwóźdź-Lukawska and Jachymski (J. Math. Anal. Appl. 356:453-463, 2009) by using the context of metric spaces endowed with a graph. The purpose of this article is to present some new fixed point results for graphic contractions and for Ćirić-Reich-Rus G-contractions on complete metric spaces endowed with a graph. The particular case of almost contractions is also considered.
\end{abstract}

MSC: $47 \mathrm{H} 10 ; 54 \mathrm{H} 25$

Keywords: metric space; connected graph; fixed point; graphic contraction; almost contraction; generalized contraction

\section{Introduction}

A very interesting approach in the theory of fixed points in some general structures was recently given by Jachymski [1] and Gwóźdź-Lukawska and Jachymski [2] by using the context of metric spaces endowed with a graph. More precisely, let $(X, d)$ be a metric space and $\Delta$ be the diagonal of $X \times X$. Let $G$ be a directed graph such that the set $V(G)$ of its vertices coincides with $X$ and $\Delta \subseteq E(G)$, where $E(G)$ is the set of edges of the graph. Assume also that $G$ has no parallel edges, and thus, one can identify $G$ with the pair $(V(G), E(G))$.

By definition, an operator $f: X \rightarrow X$ is called a Banach $G$-contraction (see Definition 2.1 in Jachymski [1]) if and only if:

(a) for each $x, y \in X$ with $(x, y) \in E(G)$, we have $(f(x), f(y)) \in E(G)$;

(b) there exists $\alpha \in] 0,1[$ such that for each $x, y \in X$, the following implication holds: $((x, y) \in E(G)$ implies $d(f(x), f(y)) \leq \alpha d(x, y))$.

If $x$ and $y$ are vertices of $G$, then a path in $G$ from $x$ to $y$ of length $k \in \mathbb{N}$ is a finite sequence $\left(x_{n}\right)_{n \in\{0,1,2, \ldots, k\}}$ of vertices such that $x_{0}=x, x_{k}=y$ and $\left(x_{i-1}, x_{i}\right) \in E(G)$ for $i \in\{1,2, \ldots, k\}$. Notice that a graph $G$ is connected if there is a path between any two vertices, and it is weakly connected if $\tilde{G}$ is connected, where $\tilde{G}$ denotes the undirected graph obtained from $G$ by ignoring the direction of edges.

Denote by $G^{-1}$ the graph obtained from $G$ by reversing the direction of edges. Thus,

$$
E\left(G^{-1}\right)=\{(x, y) \in X \times X:(y, x) \in E(G)\} .
$$

O 2012 Chifu and Petrușel; licensee Springer. This is an Open Access article distributed under the terms of the Creative Commons Attribution License (http://creativecommons.org/licenses/by/2.0), which permits unrestricted use, distribution, and reproduction in any medium, provided the original work is properly cited. 
Since it is more convenient to treat $\tilde{G}$ as a directed graph for which the set of its edges is symmetric, under this convention, we have that

$$
E(\tilde{G})=E(G) \cup E\left(G^{-1}\right) .
$$

If $G$ is such that $E(G)$ is symmetric, then for $x \in V(G)$, the symbol $[x]_{G}$ denotes the equivalence class of the relation $\Re$ defined on $V(G)$ by the rule

$y \Re z$ if there is a path in $G$ from $y$ to $z$.

Recall that if $f: X \rightarrow X$ is an operator, then by

$$
F_{f}:=\{x \in X: x=f(x)\}
$$

we denote the set of all fixed points of $f$. Denote also

$$
X_{f}:=\{x \in X:(x, f(x)) \in E(G)\} .
$$

The operator $f: X \rightarrow X$ is called orbitally continuous if for all $x, a \in X$, for any sequence $(n(i))_{i \in \mathbb{N}}$ of positive integers,

$$
\lim _{i \rightarrow \infty} f^{n(i)}(x)=a \text { implies } \quad \lim _{i \rightarrow \infty} f\left(f^{n(i)}(x)\right)=f(a) .
$$

One of the main results in Jachymski [1] is Theorem 3.2. We present here (partially) this result. In this theorem, as well as in other results of this type, one uses the following property:

for any sequence $\left(x_{n}\right)_{n \in \mathbb{N}} \subset X$, if $x_{n} \rightarrow x$ as $n \rightarrow+\infty$ and $\left(x_{n}, x_{n+1}\right) \in E(G)$ (or respectively $\left.\left(x_{n+1}, x_{n}\right) \in E(G)\right)$ for each $n \in \mathbb{N}$, then there exists a sub-sequence $\left(x_{k_{n}}\right)_{n \in \mathbb{N}}$ of $\left(x_{n}\right)_{n \in \mathbb{N}}$ such that $\left(x_{k_{n}}, x\right) \in E(G)$ (or respectively $\left.\left(x, x_{k_{n}}\right) \in E(G)\right)$ for each $n \in \mathbb{N}$.

Theorem 1.3 (Jachymski [1]) Let $(X, d)$ be a complete metric space, and let $G$ be a directed graph such that $V(G)=X, \Delta \subseteq E(G)$ and the triple $(X, d, G)$ has the property $(\mathrm{P})$. Let $f$ : $X \rightarrow X$ be a G-contraction. Then the following statements hold:

(1) $F_{f} \neq \emptyset$ if and only if $X_{f} \neq \emptyset$;

(2) if $X_{f} \neq \emptyset$ and $G$ is weakly connected, then $f$ is a Picard operator, i.e., $F_{f}=\left\{x^{*}\right\}$ and $\left(f^{n}(x)\right)_{n \in \mathbb{N}} \rightarrow x^{*}$ as $n \rightarrow \infty$ for all $x \in X$;

(3) for any $x \in X_{f}$, we have that $\left.f\right|_{[x]_{\tilde{G}}}$ is a Picard operator;

(4) if $f \subseteq E(G)$, then $f$ is a weakly Picard operator, i.e., $F_{f} \neq \emptyset$ and, for each $x \in X$, we have $\left(f^{n}(x)\right)_{n \in \mathbb{N}} \rightarrow x^{\prime \prime}(x) \in F_{f}$ as $n \rightarrow \infty$.

The purpose of this paper is to extend the above result to the case of some generalized contractions. More precisely, the cases of Ćirić-Reich-Rus G-contractions, G-graphic contractions and of $G$-almost contractions in the sense of Berinde will be considered. As one can see from the recent literature (see [3-6]), these classes of operators are important extensions of the classical contraction mapping. 
Our results also generalize and extend some fixed point theorems in partially ordered complete metric spaces given in Harjani and Sadarangani [7], Nicolae, O'Regan and Petruşel [8], Nieto and Rodríguez-López [9] and [10], Agarwal, El-Gebeily and O’Regan [16], Nieto, Pouso and Rodríguez-López [11], O’Regan and Petruşel [12], Petruşel and Rus [13], and Ran and Reurings [14].

\section{Main results}

Let $G$ be a directed graph. Throughout this section, we will suppose that the graph $G$ satisfies the following standard conditions: the set $V(G)$ of its vertices coincides with $X$, $E(G)$ is the set of edges of the graph, and $\Delta$ (the diagonal of $X \times X$ ) is a subset of $E(G)$. We also denote

$$
X^{f}:=\{x \in X:(x, f(x)) \in E(G) \text { or }(f(x), x) \in E(G)\} .
$$

Definition 2.1 The mapping $f: X \rightarrow X$ is a G-graphic contraction if:

(1) $f$ is edge preserving, i.e., $(x, y) \in E(G)$ implies $(f(x), f(y)) \in E(G)$;

(2) there exists $a \in[0,1[$ such that

$$
d\left(f(x), f^{2}(x)\right) \leq a d(x, f(x)) \text { for all } x \in X^{f} .
$$

Lemma 2.1 Let $(X, d)$ be a metric space endowed with a graph $G$ satisfying the above standard conditions. Let $f: X \rightarrow X$ be a G-graphic contraction. Then $f$ is a $G^{-1}$-graphic contraction too.

Proof Suppose that $f: X \rightarrow X$ is a G-graphic contraction. We have to prove that $f: X \rightarrow X$ is a $G^{-1}$-graphic contraction, i.e.,

(1) $(x, y) \in E\left(G^{-1}\right)$ implies $(f(x), f(y)) \in E\left(G^{-1}\right)$;

(indeed, $(x, y) \in E\left(G^{-1}\right)$ implies $(y, x) \in E(G)$. Thus, $(f(y), f(x)) \in E(G)$ and hence $(f(x)$, $f(y)) \in E\left(G^{-1}\right)$.

(2) there exists $a \in\left[0,1\left[\right.\right.$ such that $d\left(f(x), f^{2}(x)\right) \leq a d(x, f(x))$ for all $x \in X$ with $(x, f(x)) \in E\left(G^{-1}\right)$ or $(f(x), x) \in E\left(G^{-1}\right)$.

(indeed, let $x \in X$ such that $(x, f(x)) \in E\left(G^{-1}\right)$ or $(f(x), x) \in E\left(G^{-1}\right)$. Then $(f(x), x) \in E(G)$ or $(x, f(x)) \in E(G)$. In both cases, we get, by the $G$-graphic contraction condition, that there exists $a \in\left[0,1\left[\right.\right.$ such that $d\left(f(x), f^{2}(x)\right) \leq a d(x, f(x))$.

Notice now the fact that a G-graphic contraction is also a $\tilde{G}$-graphic contraction. This follows by $(*),(* *)$, the symmetry of the metric $d$ and the above lemma.

Definition 2.2 The operator $f: X \rightarrow X$ is called a Ćirić-Reich-Rus G-contraction if and only if:

(a) $f$ is edge preserving, i.e. for each $x, y \in X$ with $(x, y) \in E(G)$ we have that $(f(x), f(y)) \in E(G)$

(b) there exists $\alpha, \beta, \gamma \in \mathbb{R}_{+}$with $\left.\alpha+\beta+\gamma \in\right] 0,1[$ such that for each $x, y \in X$, we have $((x, y) \in E(G)$ implies $d(f(x), f(y)) \leq \alpha d(x, y)+\beta d(x, f(x))+\gamma d(y, f(y)))$.

In particular, if $\beta=\gamma=0$, then we get the concept of Banach $G$-contraction presented in the first section.

A similar result to the above one (see Lemma 2.1) is the following. 
Lemma 2.2 Let $(X, d)$ be a metric space endowed with a graph $G$ satisfying the standard conditions mentioned at the beginning of this section. Let $f: X \rightarrow X$ be a Cirić-Reich-Rus G-contraction. Then $f$ is a Cirić-Reich-Rus $G^{-1}$-contraction too.

Notice also that a Ćirić-Reich-Rus G-contraction is a Ćirić-Reich-Rus $\tilde{G}$-contraction.

Now, we can prove the main results of this paper. We first present some auxiliary results.

Lemma 2.3 Let $(X, d)$ be a metric space endowed with a graph $G$ satisfying the above standard conditions. Let $f: X \rightarrow X$ be a G-graphic contraction with the constant a. Then, given $x \in X^{f}$, there exists $r(x) \geq 0$ such that

$$
d\left(f^{n}(x), f^{n+1}(x)\right) \leq a^{n} r(x), \quad \text { for all } n \in \mathbb{N} .
$$

Proof Let $x \in X^{f}$, i.e., $(x, f(x)) \in E(G)$ or $(f(x), x) \in E(G)$. If $(x, f(x)) \in E(G)$, then by induction, we have that $\left(f^{n}(x), f^{n+1}(x)\right) \in E(G)$ for each $n \in \mathbb{N}$. Thus

$$
d\left(f^{n}(x), f^{n+1}(x)\right) \leq a d\left(f^{n-1}(x), f^{n}(x)\right) \leq \cdots \leq a^{n} d(x, f(x)):=a^{n} r(x) .
$$

If $(f(x), x) \in E(G)$, again by induction, we have that $\left(f^{n+1}(x), f^{n}(x)\right) \in E(G)$ for each $n \in \mathbb{N}$. Thus

$$
d\left(f^{n}(x), f^{n+1}(x)\right) \leq a d\left(f^{n-1}(x), f^{n}(x)\right) \leq \cdots \leq a^{n} d(x, f(x)):=a^{n} r(x)
$$

Lemma 2.4 Let $(X, d)$ be a complete metric space endowed with a graph $G$ satisfying the above standard conditions. Suppose that $f: X \rightarrow X$ is a G-graphic contraction. Then for each $x \in X^{f}$, there exists $x^{\prime \prime}(x) \in X$ such that the sequence $\left(f^{n}(x)\right)_{n \in \mathbb{N}}$ converges to $x^{\prime \prime}(x)$ as $n \rightarrow+\infty$.

Proof Let $x \in X^{f}$. By Lemma 2.3, we get that

$$
d\left(f^{n}(x), f^{n+1}(x)\right) \leq a^{n} r(x), \quad \text { for all } n \in \mathbb{N},
$$

where $r(x):=d(x, f(x))$. Hence $\sum_{n \geq 0} d\left(f^{n}(x), f^{n+1}(x)\right)<\infty$, proving by a standard argument that the sequence $\left(f^{n}(x)\right)_{n \in \mathbb{N}}$ is Cauchy. Since the space $X$ is complete with respect to $d$, we get that there exists $x^{*}(x) \in X$ such that the sequence $\left(f^{n}(x)\right)_{n \in \mathbb{N}}$ converges to $x^{\prime \prime}(x)$ as $n \rightarrow+\infty$.

The next result is also important.

Lemma 2.5 Let $(X, d)$ be a metric space endowed with a graph $G$ satisfying the above standard conditions. Let $f: X \rightarrow X$ be a G-graphic contraction for which there exists $x_{0} \in X$ such that $f\left(x_{0}\right) \in\left[x_{0}\right]_{\tilde{G}}$. Then the set $\left[x_{0}\right]_{\tilde{G}}$ is invariant with respect to $f$ and $\left.f\right|_{\left[x_{0}\right]_{\tilde{G}}}$ is a $\tilde{G}_{x_{0}}$-graphic contraction, where $\tilde{G}_{x_{0}}$ is the component of $\tilde{G}$ containing $x_{0}$.

Proof Let $x \in\left[x_{0}\right]_{\tilde{G}}$. Then there exist $\left(x_{0}, x_{1}, \ldots, x_{k-1}, x_{k}=x\right)$ which form a path between $x_{0}$ and $x$ in $\tilde{G}$, i.e., $\left(x_{i}, x_{i+1}\right) \in E(\tilde{G})$ for $i \in\{0, \ldots, k-1\}$. Since $f$ is a $\tilde{G}$-graphic contraction (thus edge preserving), we get that $\left(f\left(x_{i}\right), f\left(x_{i+1}\right)\right) \in E(\tilde{G})$ for $i \in\{0, \ldots, k-1\}$, i.e., we have 
a path from $f\left(x_{0}\right)$ to $f(x)$. Hence $f(x) \in\left[f\left(x_{0}\right)\right]_{\tilde{G}}=\left[x_{0}\right]_{\tilde{G}}$ since $f\left(x_{0}\right) \in\left[x_{0}\right]_{\tilde{G}}$. Thus, the set $\left[x_{0}\right]_{\tilde{G}}$ is invariant with respect to $f$.

Let $(x, y) \in E\left(\tilde{G}_{x_{0}}\right)$. Then there is a path from $x_{0}$ to $y$ passing through $x$, i.e., $\left(x_{0}, x_{1}, \ldots\right.$, $\left.x_{k-1}=x, x_{k}=y\right)$ and $\left(x_{i}, x_{i+1}\right) \in E(\tilde{G})$ for $i \in\{0, \ldots, k-1\}$. As before, by the edge preserving condition, we get that $\left(f\left(x_{i}\right), f\left(x_{i+1}\right)\right) \in E(\tilde{G})$ for $i \in\{0, \ldots, k-1\}$. Let $\left(y_{0}, y_{1}, \ldots, y_{l-1}, y_{l}\right)$ be a path between $x_{0}$ and $f_{x_{0}}$. Thus

$$
\left(x_{0}=y_{0}, y_{1}, \ldots, y_{l}=f\left(x_{0}\right), f\left(x_{1}\right), \ldots, f\left(x_{k-1}\right)=f(x), f\left(x_{k}\right)=f(y)\right)
$$

is a path in $\tilde{G}$ from $x_{0}$ to $f(y)$ such that $(f(x), f(y)) \in E\left(\tilde{G_{x_{0}}}\right)$. Moreover, $f$ is a $\tilde{G}_{x_{0}}$-graphic contraction since $E\left(\tilde{G}_{x_{0}}\right) \subset E(\tilde{G})$, and $f$ is a $\tilde{G}$-graphic contraction.

For our next result, we need the following definition.

Definition 2.3 Let $(X, d)$ be a metric space endowed with a graph $G$ satisfying the above standard conditions. The operator $f: X \rightarrow X$ is called orbitally $G$-continuous if, for all $x, a \in X$ and for any sequence $(n(i))_{i \in \mathbb{N}}$ of positive integers,

$$
\lim _{i \rightarrow \infty} f^{n(i)}(x)=a, \quad\left(f^{n(i)}(x), f^{n(i)+1}(x)\right) \in E(G), \quad i \in \mathbb{N} \quad \Rightarrow \quad \lim _{i \rightarrow \infty} f\left(f^{n(i)}(x)\right)=f(a) .
$$

We can now give the main result for $G$-graphic contractions. We will make use of the property (P) introduced in Section 1.

Theorem 2.1 Let $(X, d)$ be a complete metric space and $G$ be a directed graph satisfying the above standard conditions such that the triple $(X, d, G)$ satisfies the property $(\mathrm{P})$. Let $f: X \rightarrow X$ be a G-graphic contraction which is G-orbitally continuous. Then the following statements hold:

(1) $F_{f} \neq \emptyset$ if and only if $X^{f} \neq \emptyset$;

(2) if $X^{f} \neq \emptyset$ and $G$ is weakly connected, then $f$ is a weakly Picard operator;

(3) for any $x \in X^{f}$, we have that $\left.f\right|_{[x]_{\tilde{G}}}$ is a weakly Picard operator.

Proof We start by proving statement (3). Let $x \in X^{f}$. Applying Lemma 2.3, we have that there exists $r(x) \geq 0$ such that

$$
d\left(f^{n}(x), f^{n+1}(x)\right) \leq a^{n} r(x), \quad \text { for all } n \in \mathbb{N} .
$$

This yields, as in the proof of Lemma 2.4, that there exists $x^{*}(x) \in X$ such that $\lim _{n \rightarrow \infty} f^{n}(x)=x^{\prime \prime}(x)$. Because $x \in X^{f}$, condition (1) in Definition 2.1 implies that $f^{n}(x) \in X^{f}$ for every $n \in \mathbb{N}$. Suppose now, for example, that $(x, f(x)) \in E(G)$. (A similar deduction can be made if $(f(x), x) \in E(G)$.) Using the property (P), we can find a subsequence $\left(f^{k_{n}}(x)\right)_{n \in \mathbb{N}}$ of $\left(f^{n}(x)\right)_{n \in \mathbb{N}}$ such that $\left(f^{k_{n}}(x), x^{*}(x)\right) \in E(G)$ for each $n \in \mathbb{N}$. Then the points $x, f(x), \ldots, f^{k_{1}}(x), x^{*}(x)$ form a path in $G$, and thus $x^{\prime \prime}(x) \in[x]_{\tilde{G}}$. Since $f$ is $G$-orbitally continuous, we obtain that $x^{*}(x)$ is a fixed point for $\left.f\right|_{[x]}$.

For (1), notice that from (3) it follows that $F_{f} \neq \emptyset$ if $X^{f} \neq \emptyset$. Suppose $F_{f} \neq \emptyset$. Since we work under the assumption that $\Delta \subseteq E(G)$, we immediately get that $X^{f} \neq \emptyset$. Hence, (1) also holds. 
To prove (2), let $x \in X^{f}$. Because $G$ is weakly connected, we have that $X=[x]_{\tilde{G}}$ and we only need to apply (3).

Remark 2.1 In the above result, we can remove the condition that the triple $(X, d, G)$ satisfies the property (P) and $f$ is $G$-orbitally continuous if we assume that the mapping $f$ is orbitally continuous.

Remark 2.2 If we relax the definition of the G-graphic contraction, by replacing the condition

(1) $f$ is edge preserving, i.e., $(x, y) \in E(G)$ implies $(f(x), f(y)) \in E(G)$

with

$\left(1^{\prime}\right)(x, f(x)) \in E(G)$ implies $\left(f(x), f^{2}(x)\right) \in E(G)$,

then part of the above results take place. More precisely, we do not know if a similar result to Lemma 2.5 takes place.

The following example shows that our result is a non-trivial extension of the above mentioned papers.

Example 2.1 Let $X=[0,1]$ be endowed with the usual distance. Consider

$$
E(G)=\{(0,0)\} \cup\{(0, x): x \geq 1 / 2\} \cup\{(x, y): x, y \in(0,1]\},
$$

and $f: X \rightarrow X$,

$$
f(x)= \begin{cases}x / 2, & \text { if } x \in(0,1) \\ 3 / 4, & \text { if } x=0 \\ 1, & \text { if } x=1\end{cases}
$$

Then $G$ is weakly connected, $X^{f}$ is nonempty and $f$ is a $G$-graphic contraction which fails to be a $G$-contraction. Moreover, $\operatorname{Fix}(f)=\{1\}$.

Proof It can be easily checked that $G$ is weakly connected and $f$ is edge preserving. To see that $f$ is not a $G$-contraction, notice that $|f(0)-f(1 / 2)|=1 / 2=|0-1 / 2|$. A simple case-bycase calculation shows that $f$ is a G-graphic contraction. Moreover, Fix $(f)=\{1\}$.

We will consider next the case of almost contractions; see Berinde [3]. See also Păcurar [15] for a detailed and comprehensive study of almost contractions.

Definition 2.4 The mapping $f: X \rightarrow X$ is a $G$-almost contraction if:

(1) $f$ is edge preserving, i.e., $(x, y) \in E(G)$ implies $(f(x), f(y)) \in E(G)$;

(2) there exists $\alpha \in] 0,1[$ and $L \geq 0$ such that for each $x, y \in X$ the following implication holds:

$$
(x, y) \in E(G) \quad \text { implies } \quad d(f(x), f(y)) \leq a d(x, y)+L d(y, f(x)) .
$$


Notice that if $f$ is a $G$-almost contraction, then $f$ is a G-graphic contraction with the constant $a \in[0,1[$. Thus all the above results take place.

We will consider now the case of Ćirić-Reich-Rus G-contractions.

Lemma 2.6 Let $(X, d)$ be a metric space endowed with a graph $G$ satisfying the above standard conditions. Let $f: X \rightarrow X$ be a Cirić-Reich-Rus G-contraction with constants $\alpha, \beta, \gamma$. Then, given $x \in X^{f}$, there exists $r(x) \geq 0$ such that

$$
d\left(f^{n}(x), f^{n+1}(x)\right) \leq a^{n} r(x), \quad \text { for all } n \in \mathbb{N}
$$

where $a:=\frac{\alpha+\beta}{1-\gamma}$.

Proof Let $x \in X^{f}$, i.e., $(x, f(x)) \in E(G)$ or $(f(x), x) \in E(G)$. If $(x, f(x)) \in E(G)$, then by induction, we have that $\left(f^{n}(x), f^{n+1}(x)\right) \in E(G)$ for each $n \in \mathbb{N}$. Hence

$$
d\left(f^{n}(x), f^{n+1}(x)\right) \leq \alpha d\left(f^{n-1}(x), f^{n}(x)\right)+\beta d\left(f^{n-1}(x), f^{n}(x)\right)+\gamma d\left(f^{n}(x), f^{n+1}(x)\right) .
$$

Thus, we get that

$$
d\left(f^{n}(x), f^{n+1}(x)\right) \leq \frac{\alpha+\beta}{1-\gamma} d\left(f^{n-1}(x), f^{n}(x)\right) \leq \cdots \leq a^{n} d(x, f(x)):=a^{n} r(x) .
$$

If $(f(x), x) \in E(G)$, again by induction, we have that $\left(f^{n+1}(x), f^{n}(x)\right) \in E(G)$ for each $n \in \mathbb{N}$. Thus we get the same relation as before. This completes the proof.

Lemma 2.7 Let $(X, d)$ be a complete metric space endowed with a graph $G$ satisfying the above standard conditions. Suppose that $f: X \rightarrow X$ is a Cirić-Reich-Rus G-contraction with constants $\alpha, \beta, \gamma$. Then for each $x \in X^{f}$, there exists $x^{*}(x) \in X$ such that the sequence $\left(f^{n}(x)\right)_{n \in \mathbb{N}}$ converges to $x^{*}(x)$ as $n \rightarrow+\infty$.

Proof Let $x \in X^{f}$. By Lemma 2.6, we get that

$$
d\left(f^{n}(x), f^{n+1}(x)\right) \leq a^{n} r(x), \quad \text { for all } n \in \mathbb{N}
$$

where $r(x):=d(x, f(x))$. Hence $\sum_{n \geq 0} d\left(f^{n}(x), f^{n+1}(x)\right)<\infty$, proving by a standard argument that the sequence $\left(f^{n}(x)\right)_{n \in \mathbb{N}}$ is Cauchy. Since the space $X$ is complete with respect to $d$, we get that there exists $x^{\prime \prime}(x) \in X$ such that the sequence $\left(f^{n}(x)\right)_{n \in \mathbb{N}}$ converges to $x^{\prime \prime}(x)$ as $n \rightarrow+\infty$.

We can now give the main result for Ćirić-Reich-Rus G-contractions. We will make use again of the property $(\mathrm{P})$ introduced in Section 1.

Theorem 2.2 Let $(X, d)$ be a complete metric space and $G$ be a directed graph satisfying the above standard conditions such that the triple $(X, d, G)$ satisfies the property $(\mathrm{P})$. Let $f$ : $X \rightarrow X$ be Cirić-Reich-Rus G-contraction with constants $\alpha, \beta, \gamma$ such that it is G-orbitally continuous. Then the following statements hold:

(1) $F_{f} \neq \emptyset$ if and only if $X^{f} \neq \emptyset$;

(2) if $X^{f} \neq \emptyset$ and $G$ is weakly connected, then $f$ is a weakly Picard operator;

(3) for any $x \in X^{f}$, we have that $\left.f\right|_{[x]_{\tilde{G}}}$ is a weakly Picard operator. 
Proof We start by proving statement (3). Let $x \in X^{f}$. Applying Lemma 2.6, we have that there exists $r(x) \geq 0$ such that

$$
d\left(f^{n}(x), f^{n+1}(x)\right) \leq a^{n} r(x), \quad \text { for all } n \in \mathbb{N} .
$$

This yields, as in the proof of Lemma 2.7, that there exists $x^{*}(x) \in X$ such that $\lim _{n \rightarrow \infty} f^{n}(x)=x^{*}(x)$. Because $x \in X^{f}$, condition (1) in Definition 2.2 implies that $f^{n}(x) \in X^{f}$ for every $n \in \mathbb{N}$. Suppose now, for example, that $(x, f(x)) \in E(G)$. (A similar deduction can be made if $(f(x), x) \in E(G)$.) Using the property (P), we can find a subsequence $\left(f^{k_{n}}(x)\right)_{n \in \mathbb{N}}$ of $\left(f^{n}(x)\right)_{n \in \mathbb{N}}$ such that $\left(f^{k_{n}}(x), x^{*}(x)\right) \in E(G)$ for each $n \in \mathbb{N}$. Then the points $x, f(x), \ldots, f^{k_{1}}(x), x^{*}(x)$ form a path in $G$, and thus $x^{*}(x) \in[x]_{\tilde{G}}$. Since $f$ is $G$-orbitally continuous, we obtain that $x^{*}(x)$ is a fixed point for $\left.f\right|_{[x]}$.

For (1), notice that from (3) it follows that $F_{f} \neq \emptyset$ if $X^{f} \neq \emptyset$. Suppose $F_{f} \neq \emptyset$. Since we work under the assumption that $\Delta \subseteq E(G)$, we immediately get that $X^{f} \neq \emptyset$. Hence, (1) also holds.

To prove (2), let $x \in X^{f}$. Because $G$ is weakly connected, we have that $X=[x]_{\tilde{G}}$, and we only need to apply (3).

Remark 2.3 If in the above result we put $\beta=\gamma=0$, then we get the results given in Jachymski [1].

Remark 2.4 It is an open problem if in some appropriate assumptions, one can prove that a Ćirić-Reich-Rus G-contraction is a Picard operator.

\section{Competing interests}

The authors declare that they have no competing interests.

\section{Authors' contributions}

Both authors had have equal contribution to this article regarding results, conception and drafting the manuscript. The authors read and approved the final manuscript.

\section{Received: 27 March 2012 Accepted: 7 September 2012 Published: 25 September 2012}

\section{References}

1. Jachymski, J: The contraction principle for mappings on a metric space with a graph. Proc. Am. Math. Soc. 136, 1359-1373 (2008)

2. Gwóźdź-Lukawska, G, Jachymski, J: IFS on a metric space with a graph structure and extensions of the Kelisky-Rivlin theorem. J. Math. Anal. Appl. 356, 453-463 (2009)

3. Berinde, $\mathrm{V}$ : Approximating fixed points of weak contractions using the Picard iteration. Nonlinear Anal. Forum 9 43-53 (2004)

4. Berinde, V: Some remarks on a fixed point theorem for Ćirić-type almost contractions. Carpath. J. Math. 25(2), 157-162 (2009)

5. Rus, IA: Picard operators and applications. Sci. Math. Jpn. 58, 191-219 (2003)

6. Rus, IA, Petruşel, A, Petruşel, G: Fixed Point Theory. Cluj University Press, Cluj-Napoca (2008)

7. Harjani, J, Sadarangani, K: Fixed point theorems for weakly contractive mappings in partially ordered sets. Nonlinear Anal. 71, 3403-3410 (2009)

8. Nicolae, A, O'Regan, D, Petruşel, A: Fixed point theorems for singlevalued and multivalued generalized contractions in metric spaces endowed with a graph. Georgian Math. J. 18, 307-327 (2011)

9. Nieto, JJ, Rodríguez-López, R: Contractive mapping theorems in partially ordered sets and applications to ordinary differential equations. Order 22, 223-239 (2005)

10. Nieto, JJ, Rodríquez-López, R: Existence and uniqueness of fixed points in partially ordered sets and applications to ordinary differential equations. Acta Math. Sin. Engl. Ser. 23, 2205-2212 (2007)

11. Nieto, JJ, Pouso, RL, Rodríguez-López, R: Fixed point theorems in ordered abstract spaces. Proc. Am. Math. Soc. 135 2505-2517 (2007)

12. O'Regan, D, Petruşel, A: Fixed point theorems in ordered metric spaces. J. Math. Anal. Appl. 341, 1241-1252 (2008)

13. Petruşel, A, Rus, IA: Fixed point theorems in ordered L-spaces. Proc. Am. Math. Soc. 134, 411-418 (2006) 
14. Ran, ACM, Reurings, MC: A fixed point theorem in partially ordered sets and some applications to matrix equations Proc. Am. Math. Soc. 132, 1435-1443 (2004)

15. Păcurar, M: Iterative Methods for Fixed Point Approximations. Risoprint, Cluj-Napoca (2010)

16. Agarwal, RP, El-Gebeily, MA, O'Regan, D: Generalized contractions in partially ordered metric spaces. Appl. Anal. 87, 109-116 (2008)

doi:10.1186/1687-1812-2012-161

Cite this article as: Chifu and Petruşel: Generalized contractions in metric spaces endowed with a graph. Fixed Point Theory and Applications 2012 2012:161.

Submit your manuscript to a SpringerOpen ${ }^{\odot}$ journal and benefit from:

- Convenient online submission

- Rigorous peer review

- Immediate publication on acceptance

- Open access: articles freely available online

- High visibility within the field

- Retaining the copyright to your article

Submit your next manuscript at $>$ springeropen.com 